\section{Surface Preparation of Uranium by Ion Milling}

Donald A. Carpenter and Robert L. Bridges

Uranium Center of Excellence, Y-12 National Security Complex, Oak Ridge, TN

carpenterda@y12.doe.gov

\section{Introduction}

Metallographic examinations of uranium, as carried out with both optical and orientation contrast microscopies (electron backscatter diffraction or EBSD), typically require a final preparation step that involves acid-etching or electropolishing.[1][2]. Uranium oxidizes relatively rapidly after mechanical polishing, making EBSD impossible without additional preparation steps. Conventional specimen preparation generates a mixture of acids and radioactive waste, so-called "mixed waste" as defined by the Resource Conservation and Recovery Act (RCRA). Mixed waste is more costly to dispose of than either separate component and there is a desire to eliminate mixed waste streams. One simple method to avoid the use of acids in optical metallography is to wait a few minutes for the sample to differentially oxidize to obtain the desired contrast. However, this method is disadvantageous when more than a few micrographs are needed because the sample continues to change with each micrograph.
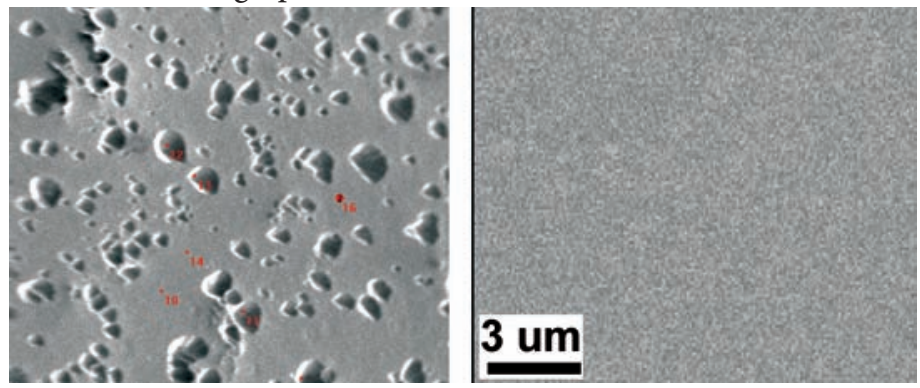
(right)

Figure 1. SEM images of ion milled surfaces. Argon (left), xenon

Another approach to the final polish or etch is ion milling. We will describe ion milling techniques for uranium surfaces for both EBSD and optical microscopy, using a broad ion-beam source that result in surfaces that are more consistent and have extended handling times.

\section{Experimental Methods}

All specimens were mounted in epoxy and mechanically ground and polished, generally using the techniques described in reference [2], with $0.05-\mu \mathrm{m}$ alumina as the final polishing medium. All optical

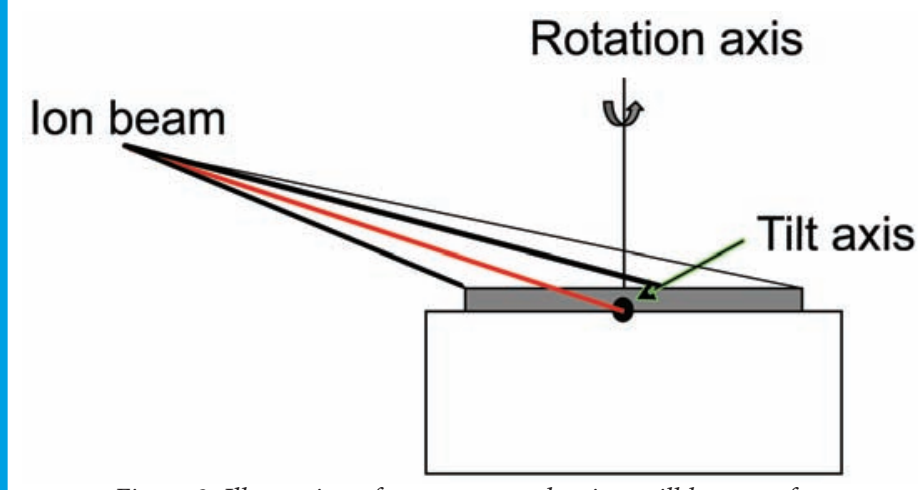

Figure 2. Illustration of geometry used to ion mill large surface area with broad beam

microscopy and some EBSD

specimens were mounted in conducting mounts (Buehler Probe-met) to provide electrical conductivity for ion milling. Those EBSD specimens in non-conducting mounts were removed from the mounts before ion milling. A commercial ion etching and coating system, the Gatan model 682, Precision Etching Coating System, was used with xenon gas as the etching and polishing species. Argon ions, typically used in ion milling applications, tended to roughen the surface by forming micron-

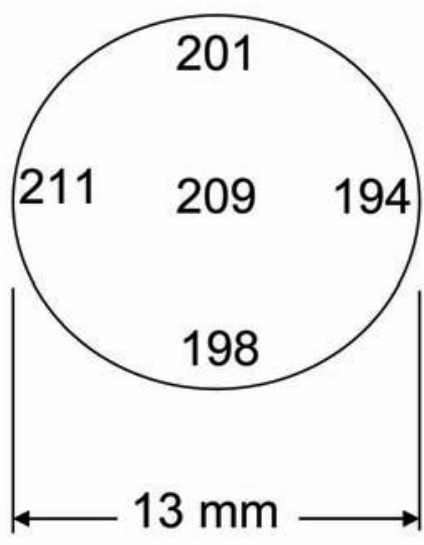

Figure 3. Average Image quality values on the surface of xenonion-milled, cast uranium indicated polishing was uniformly distributed.

sized bumps. The heavier xenon ions produced a smoother surface than argon, presumably because their greater momentum more efficiently removed uranium atoms. Figure 1 shows a comparison of the surfaces produced by the two ion beams.

Of the several commercially available ion etching and polishing instruments, most are configured so that the ion beam hits the specimen at the intersection of the tilt and rotation axes. Thus, a flat sample is placed into the instrument so that, ideally, the surface to be etched or polished contains the tilt axis and is normal to the rotation axis. Typically, the sample is rotated at a fixed tilt angle. Low tilt angles (incidence angle relative to the surface normal) remove more material, but cause more damage. High tilt angles planarize, removing less material but removing it more uniformly, for a polishing effect.

An important issue with ion milling was that the Gaussian shape of the ion beam can produce a surface where the center of the sample sees a greater number of ions than the edges. Of course, standard methods involving high incidence angles and sample rotation tended to improve homogeneity in the center region, but still left differences between center and edges in the amount of material removed. It was possible to broaden the beam by reducing the gas flow and get further improvement in homogeneity. The result of manipulating the gas flow was easily observed optically, since uranium glowed brightly under the influence of an ion beam. An effective additional step was to raise the sample surface slightly above the tilt axis so

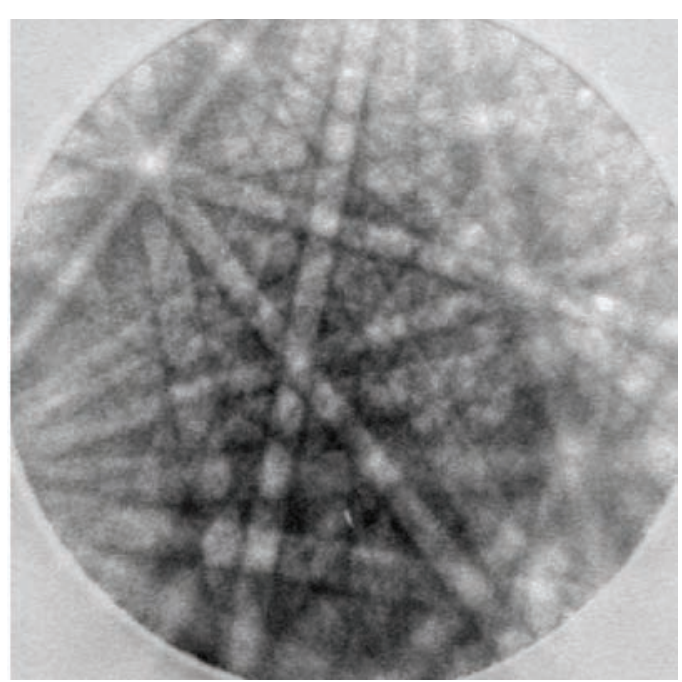

Figure 5. Typical EBSD pattern resulting from xenon-ion beam milling. (128x128) 


\section{Beating the Competition is Easy, When You've Got Products They Don't Offer!}

Si-Li EDS Detectors, IXRF offers guaranteed, premium $\leq 130 \mathrm{eV}$, resolution $\mathrm{Si}-\mathrm{Li}$ detectors. (*industry standard detector resolution $133-138 \mathrm{eV}$ ) that carry a three year warranty.

New $30 \mathrm{~mm}^{2}$ at $133 \mathrm{eV}$, three year warranty available for cost effective fast X-ray Mapping.
fX SEM XRF,

400-1000 micron spot. 10,000

times more analytically sensitive than EDS.

Perfect for environmental applications as well as non-conductive samples.

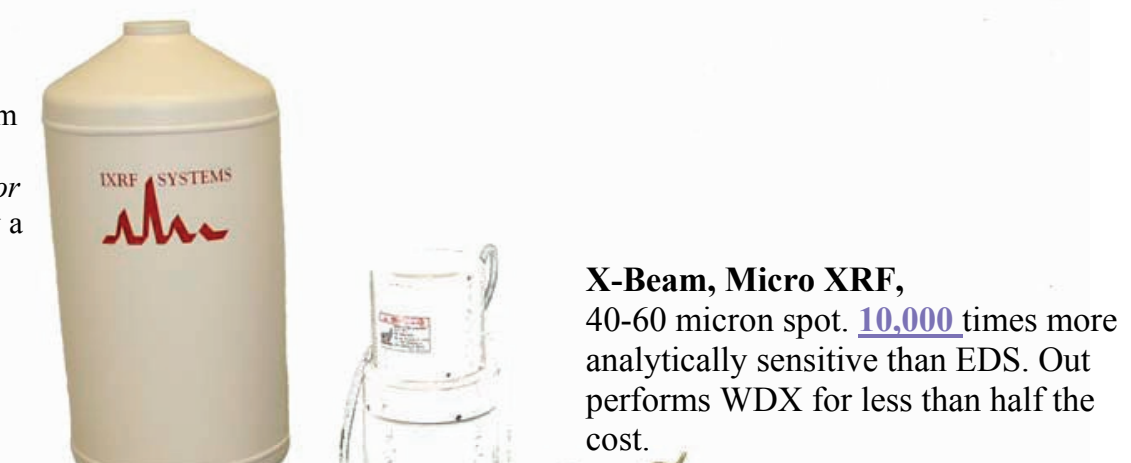

X-Beam, Micro XRF, 40-60 micron spot. 10,000 times more analytically sensitive than EDS. Out cost.

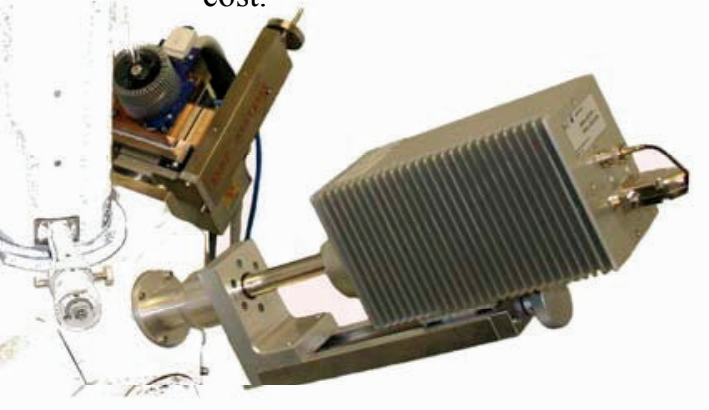

$50 \mathrm{~mm}^{2}$ Silicon Drift Detector, the largest Active Area in the industry $\left(50 \mathrm{~mm}^{2}\right) .133 \mathrm{eV}$ resolution, with optimum peak stability, and high input count rates. Who says "Bigger isn't better"?

IXRF does not stratify their software suite into low, medium, and high-end levels; there's only one high-end package that includes a myriad of Spectra Analysis, Image Analysis, X-ray

Mapping, and unsurpassed SEM/EDS Automation.

Free Software Upgrades for Life...no compromises. 


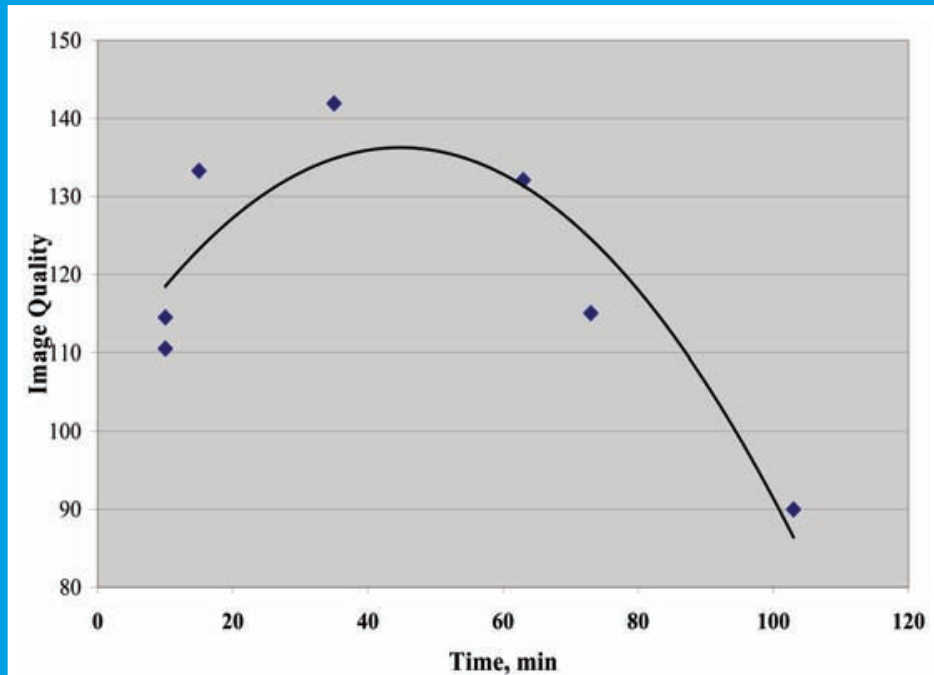

Figure 4. Effect of milling time on the IQ values of a sample of wrought uranium. Sample was mounted on copper block, tilted 70 degrees, and milled with xenon ions.

that at high tilt angles, the center of the beam was off the rotation axis. Thus, the most intense part of the beam swept out a larger area as the sample rotated. This concept is illustrated in Fig. 2. When using these techniques, average image quality (IQ) factors from OIM scans were not significantly different when measured near the edge and near the center of a round, cast uranium sample that was $13 \mathrm{~mm}$ diameter (fig. 3).

We found that handling times could be extended by applying a thin coating of carbon immediately after ion milling. The argon flow rates of the coating guns were adjusted for maximum coating rates, usually $0.2-0.4 \AA / \mathrm{s}$, with a final thickness of about $25 \AA$. That thickness had a negligible effect on the quality of the EBSD patterns.

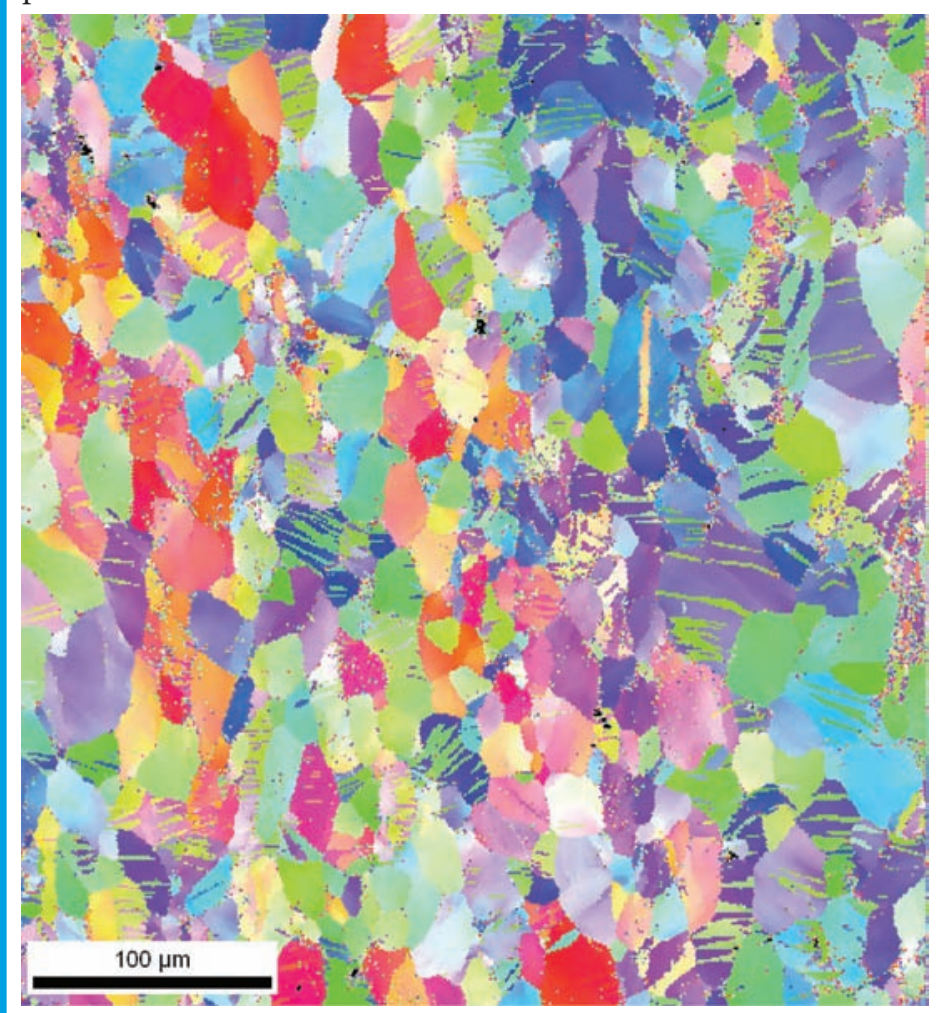

Figure 6. OIM scan of a rolled and annealed uranium specimen. Colors indicate grain orientations of the planes perpendicular to the surface normal. (30kV,1- $\mu \mathrm{m}$ step size). Scale bar is $100 \mu \mathrm{m}$.

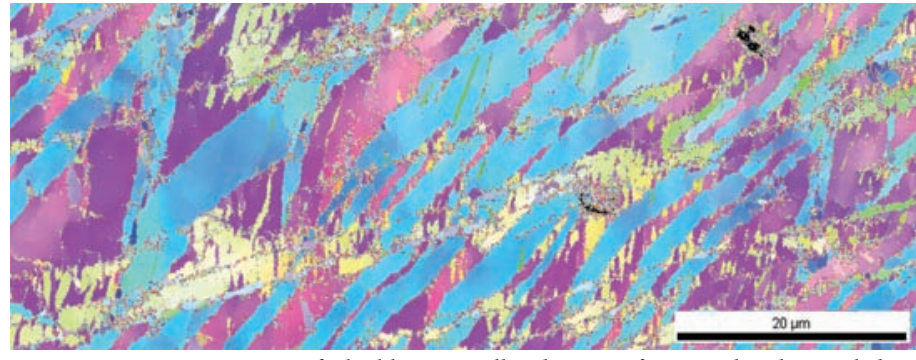

Figure 7. OIM scan of a highly recrystallized region of a swaged and annealed uranium specimen. (30kV, 0.1-mm step size). Scale bar is $20 \mathrm{~mm}$.

Since the surface requirements for EBSD and optical microscopy were different, additional ion milling steps and issues for the two microscopies will be described separately.

Ion milling of uranium for EBSD EBSD patterns arise from near-surface atoms so it was necessary to remove all oxidation and polishing damage. Even after polishing with $.05 \mu \mathrm{m}$ alumina, there was apparently enough damage to prevent the formation of EBSD patterns. It was possible to remove all visible traces of oxidation by ion milling for up to 10 minutes at $6 \mathrm{kV}$ ion-beam potential and $70^{\circ}$ tilt. However, only highly diffuse EBSD patterns could be obtained unless additional ion milling was carried out. The best surfaces obtained, so far, resulted from milling at $6 \mathrm{kV}$ and $70^{\circ}$ tilt for $30 \mathrm{~min}$., followed by two hours at $80^{\circ}$ tilt.

The metal specimen was mounted on a copper block with silver adhesive to maximize thermal conductivity. We found that if we did not control the temperature of the specimen, the heat generated during the milling process appeared to cause small surface bumps like the ones observed when argon was used (under the same conditions). No bumps were observed when the specimen was attached to the copper block and xenon was used. Note that specimens mounted in conducting mounts could be ion milled to the point where good EBSD patterns were obtained, but not without generating small bumps.

When working with un-mounted metal specimens, the best results were obtained when the specimen surface was normal to the rotation axis and raised about $1 \mathrm{~mm}$ above the surface containing the tilt axis. In lieu of an engineered leveling jig, we sometimes used a folded, thin strip of copper sheet to level a specimen with un-even

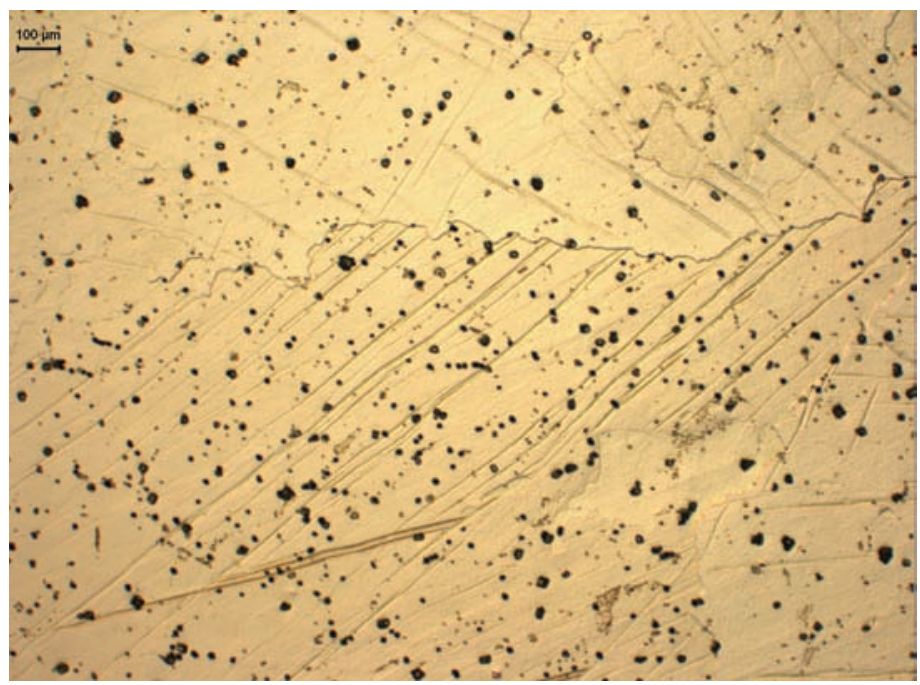

Figure 8. Bright field, DIC image of a cast uranium specimen, etched with a Xe ion beam. This specimen had been in air for one week following ion etch / carbon coating. The scale bar in the upper left corner is 100 $\mu m$. 


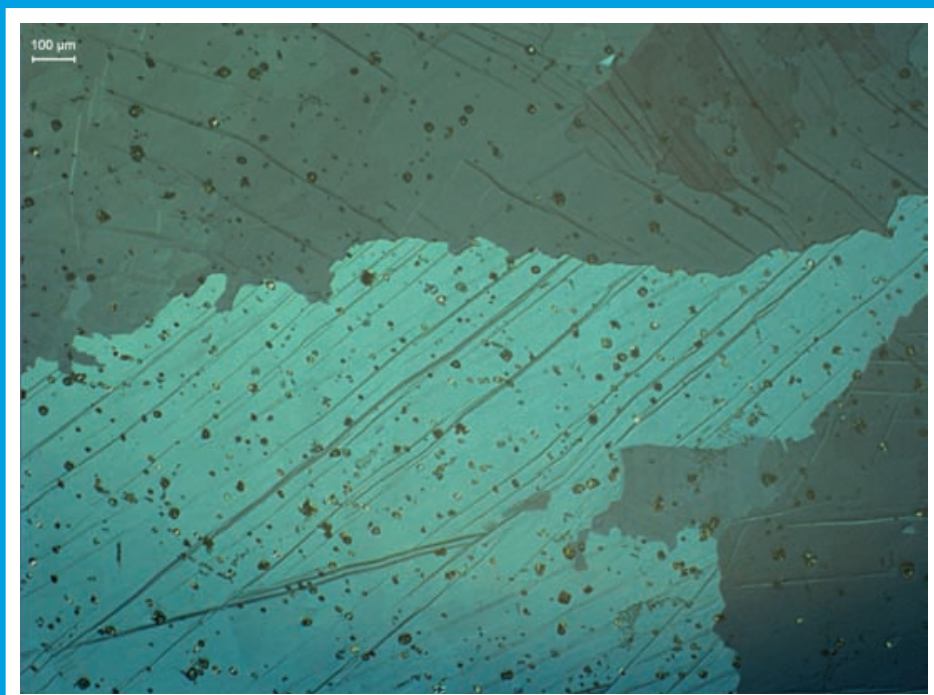

Figure 9. Polarized light image of sample in figure 8.

sides. After gluing the copper strip to the block, the low side of the specimen was glued to the folded edge and pressed down until the surface was level.

Figure 4 shows the effect of milling time on the surface quality of uranium specimens. Presumably, after about 30 minutes at $6 \mathrm{kV}$ and $70^{\circ}$ tilt, the formation of an amorphous layer began to decrease the quality of the EBSD patterns. Subsequently increasing the tilt to $80^{\circ}$ and milling for two additional hours (after $30 \mathrm{~min}$. at $70^{\circ}$ ), as recommended above, resulted in improved surfaces. A typical EBSD pattern of uranium, taken after this procedure and after coating with carbon, had excellent contrast and sharp lines (fig. 5). Examples of orientation contrast (or orientation imaging microscopy, OIM) images from ion milled uranium surfaces are given in figs. 6 and 7.

Ion milling of uranium for optical microscopy Specimens for optical microscopy were mounted in conducting mounts and ionmilled in the mounts. The primary objective for optical microscopy was to produce an etched surface. In general, a uranium surface could be adequately etched at $6 \mathrm{kV}$ by rocking the specimen between $0^{\circ}$ and $30^{\circ}$ tilt, while rotating about the surface normal, for two to five minutes. Since the surface of the mounted specimen was above the plane containing the tilt axis, the ion beam moved from center to near the edge during the rocking motion, achieving a uniform etch. After ion-beam etching, carbon was deposited, as described above. Figures 8 and 9 show optical microscopy images of a uranium surface treated as described here. The sample used for the images in figs. 8 and 9 had been left in air for one week following the ion etch / carbon coating process, before these images were made. While some oxidation was present after a week, the uniform background due to the low amount of oxidation in the bright field image of fig. 8 will permit the digitization of inclusions for automatic counting. The relative lack of handling constraints has obvious advantages in a production environment.

\section{Acknowledgement}

The Y-12 National Security Complex is managed by BWXT-Y12, L.L.C. for the U. S. Department of Energy, under contract number DE-AC05-00OR22800.

\section{References}

[1] R. J. McCabe and D. F. Teter; JOM, 232 (2006) 33.

[2] Ann M. Kelly, Dan J. Thoma, Robert D. Field, Paul S. Dunn, David F. Teter; Journal of Nuclear Materials, $\underline{353}$ (2006) 158-166

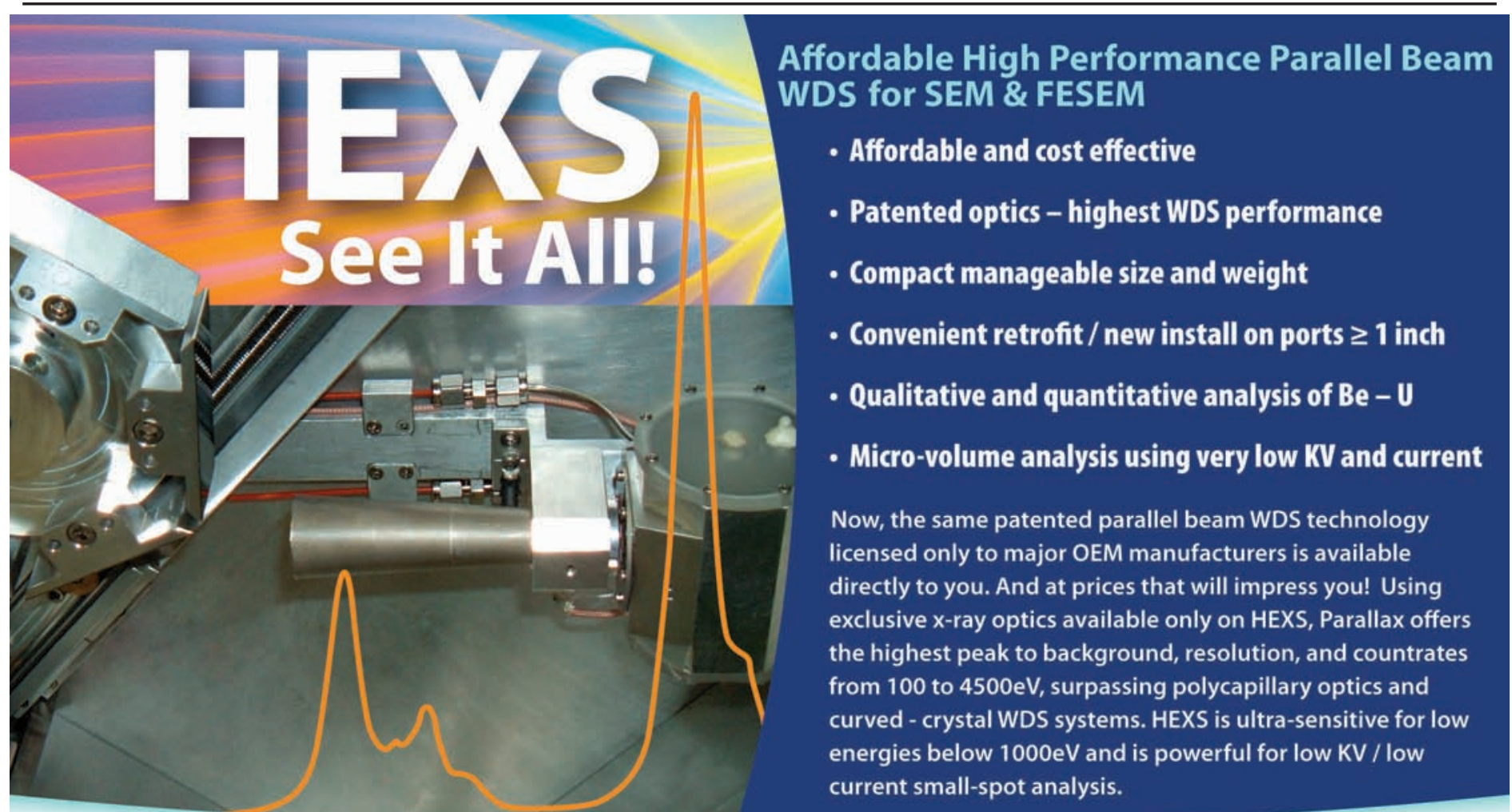

\section{HEXS. See It All! \\ Call 866.581-XRAY for more information. wWW.parallaxray.com \\ US Patents $5,768,339$ and $5,682,415$}
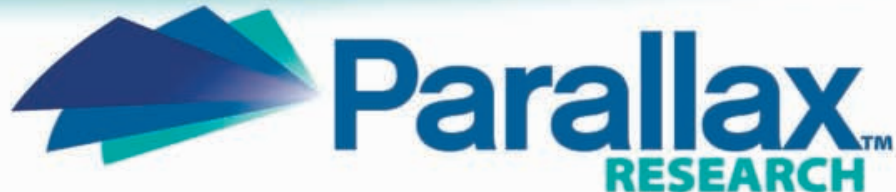